1 Secretaria de Estado de Saúde do Distrito Federal (SES-DF) - Brasília (DF), Brasil.

carol_reigada@yahoo.com.br

2 Universidade Nove de Julho (Uninove) - São

Paulo (SP), Brasil.

3 Universidade de Brasília (UnB) - Brasília (DF),

Brasil.

4 Faculdade Israelita de Ciências da Saúde Albert Einstein - São Paulo (SP), Brasil.

5 Secretaria Municipal de Saúde do Rio de Janeiro (SMS-RJ) - Rio de Janeiro (RJ), Brasil.

6 Secretaria Municipal de Saúde de Belo Horizonte (SMS-BH) - Belo Horizonte (MG), Brasil.

\section{Liderança feminina: relato do primeiro encontro de mulheres Médicas de Família e Comunidade do Brasil}

\author{
Women leadership: report of the first meeting of Female Family \\ Physicians in Brazil
}

Carolina Lopes de Lima Reigada', Denize Ornelas Pereira Salvador de Oliveira², Ana Paula Borges Carrijo3, Patrícia Sampaio Chueiri4, Julia Horita Moherdaui5, Natália Pontes de Albuquerque ${ }^{6}$

DOI: 10.1590/0103-11042021E116

\begin{abstract}
RESUMO Este relato situa-se no campo do protagonismo das mulheres Médicas de Família e Comunidade (mMFC) e em sua articulação nacional por meio do Grupo de Trabalho Mulheres na Medicina de Família e Comunidade (GT-MMF), fundado em 2016 no bojo da Sociedade de Medicina de Família e Comunidade (SBMFC), entidade científica que representa a especialidade no País. Descreve a organização do I Encontro do GT-MMFC, em 2019, intitulado 'Liderança feminina em saúde' e discute seus desdobramentos, com foco na equidade de gênero nos domínios: profissional, acadêmico, de gestão, de ensino e pesquisa; assim como na própria instituição, a SBMFC. O artigo se debruça, ainda, sobre questões relacionadas com as causas de mulheres no âmbito da especialidade e da medicina. $\mathrm{O}$ evento foi aberto a estudantes e profissionais de outras áreas e ofertou discussões contemporâneas, como: protagonismo feminino; autocuidado; interseccionalidades; maternidade e trabalho; inserção da mulher e diferenças de gênero na política. O Encontro reuniu mulheres de quatro regiões do Brasil, aprofundou as relações e o apoio interpares e permitiu a ampliação das pautas para o fortalecimento da consciência de gênero e sua influência no cotidiano das mMFC, na sua prática acadêmica, científica, assistencial e de gestão.
\end{abstract}

PALAVRAS-CHAVE Atenção Primária à Saúde. Medicina de Família e Comunidade. Mulheres. Mulheres trabalhadoras. Iniquidade de gênero.

ABSTRACT This report is situated in the scope of Female Family Physicians' protagonism and their national organization through the Women's Working Group on Family and Community Medicine, which was founded in 2016 under the Brazilian Society of Family and Community Medicine, a scientific entity that represents this medical specialty in the country. It describes the organization of the first Meeting of this Working Group, in 2019, named 'Female Leadership in Health' and discusses its unfoldings, focusing on gender equity in spheres such as: professional, academic, management, educational, and research, as well as permeating the institution itself. This article also focuses on women's issues surrounding both the specialty and medicine in general. The event welcomed students and professionals from other fields and offered contemporary debates, for example: female protagonism, self-care, intersectionality, maternity and work, participation of women in politics, and gender inequities. The Meeting gathered women from four regions of Brazil, deepened peer bonds and support, and enabled the expansion of the agenda of gender consciousness and its influence in women family physicians' daily life in their experience in management, university, science, and assistance.

KEYWORDS Primary Health Care. Family medicine. Women. Working women. Gender inequity. 


\section{Introdução}

O Grupo de Trabalho Mulheres na Medicina de Família e Comunidade (GT-MMFC) foi fundado em 2016, durante a XXI Conferência Mundial da World Organization of Family Doctors (Wonca), realizada no Rio de JaneiroRJ, quando mulheres Médicas de Família e Comunidade (mMFC), atuantes em associações estaduais e brasileira de Medicina de Família e Comunidade (MFC), articularam-se para participar das atividades do Wonca Working Party on Women \& Family Medicine (WWPWFM) ${ }^{1}$. Essas médicas consideravam que a sub-representação de mulheres nos espaços de decisão da profissão e da especialidade revela-se um problema histórico, político e de gênero. Apesar da crescente feminização da medicina ${ }^{2}$ e do debate contemporâneo sobre as iniquidades de gênero, prevalecem resquícios da dominação masculina nos campos material e simbólico, expostos por Beauvoir ${ }^{3}$ na metade do século XX.

A Conferência aproximou as mMFC e levou ao desejo coletivo de difundir o debate para âmbito nacional e ampliar seu protagonismo acadêmico e político, além da representatividade na direção e nos encontros científicos da MFC'. É importante destacar a legitimidade desse movimento a partir da própria tradição histórica da medicina, que subjugou as mulheres na sua corporificação como paciente ou no seu exercício da medicina, relegando ao masculino o lugar de prestígio e domínio do saber científico ${ }^{4}$. A construção institucional da medicina no Brasil, que marcou os séculos XIX e XX pela diferença entre os gêneros, deixa o desafio estrutural de debatê-la para subvertê-la.

O GT-MMFC, dentro da medicina e da especialidade, pretende operar na urgência de dar novos sentidos a discussões perenes. Objetiva discutir sobre a inserção das mulheres na MFC, trabalhando pela equidade de gênero nos campos de atuação profissional: academia, gestão, ensino e pesquisa; assim como na própria instituição técnico-científica da especialidade, a Sociedade Brasileira de Medicina de Família e Comunidade (SBMFC) ${ }^{5}$.

Desde sua fundação, a atuação do GT-MMFC expande-se progressivamente em várias regiões do País, com ações capilarizadas pelas mMFC, a partir do exercício em docência, pesquisa, atividades científicas da especialidade (oficinas, seminários e congressos) e representações institucionais. Nesses espaços, são pautados temas relacionados com os direitos reprodutivos e com o aborto; representatividade e protagonismo femininos; violência contra a mulher e ações interseccionais em parceria com outros GT, como o de 'Saúde da População Negra' e o de 'Sexualidade, Gênero, Diversidade e Direitos', colocando em evidência a importância de discutir racismo e interseccionalidades, além de temas contemporâneos, como a sobrecarga feminina durante a pandemia da Covid-195.

Uma ação expressiva foi a discussão, dentro da SBMFC, das Gender Equity Standards for Wonca Scientific Meetings (GES) ${ }^{6}$, uma ferramenta desenvolvida pelo WWPWFM, que estabelece parâmetros para avaliação de quanto os eventos respeitam a equidade de gênero, $\mathrm{e}$ que passou a ser utilizada no planejamento e avaliação dos encontros científicos dessa sociedade. Trata-se de uma tarefa tão exigente quanto estranha às mulheres - as iniquidades de gênero parecem demandar comprovação precisa, em uma certa cientificidade do óbvio, demarcando legitimidade e equidade ainda não garantidas entre os pares.

Durante o XV Congresso Brasileiro de Medicina de Família e Comunidade, em julho de 2019, ocorreu a reunião ordinária do GTMMFC. Nesta, surgiu a proposta do primeiro encontro nacional do GT, em espaço independente de outros eventos da SBMFC, e cujos propósitos estruturaram-se em: aprofundar o debate sobre temas como protagonismo feminino na saúde, ampliar a integração do grupo e identificar as questões prioritárias no planejamento do GT-MMFC. O encontro aconteceu ainda no mesmo ano, em Brasília (DF) ${ }^{7}$. Dessa forma, este artigo objetiva relatar e discutir a 
experiência de organização e os resultados do I Encontro do Grupo de Trabalho de Mulheres na Medicina de Família e Comunidade e sua repercussão no fortalecimento do GT-MMFC.

\section{A organização do encontro}

Inicialmente, foi estabelecida uma comissão organizadora do congresso, com mulheres de diferentes estados do Brasil. Foi proposto um formulário semiestruturado on-line para levantar os principais interesses das potenciais participantes do encontro às $\mathrm{MMFC}$ membras do GT-MMFC, de forma que a comissão priorizasse na proposta científica temas cotidianos e relevantes. Entre as 42 respostas recebidas, 18 manifestaram interesse por temas referentes à liderança feminina/síndrome da impostora; 10 levantaram o problema da violência de gênero e machismo nas consultas e nas relações de trabalho; 5 se preocupavam com a maternidade e o trabalho para as mMFC. As demais respostas complementaram com a pertinência das discussões sobre: feminismo negro, mulheres na pesquisa científica e em cargos de gestão, autocuidado e redes de colaboração entre mulheres e iniquidades de gênero nas políticas públicas. Apesar da envergadura do debate, são temas não priorizados nos eventos da SBMFC. Além disso, mais profundamente, proposições que dialogam com a luta feminista no Brasil, no enfrentamento às desigualdades na vida pública e privada, anunciando uma emancipação porventura inacabada ${ }^{8}$.

As Faculdades de Medicina e de Saúde da Universidade de Brasília (UnB) permitiram o uso de seu espaço físico, sendo disponibilizados durante três dias dois auditórios, duas salas de reunião e espaços coletivos como o jardim central e a copa. Por intermédio da Diretoria da Diversidade 9 (DIV) - eixo universitário que tem como foco representatividade, reconhecimento e visibilidade de diversidades identitárias de gênero, orientação sexual, raça e etnia -, a UnB mediou o contato com pesquisadoras e referências do Distrito Federal em áreas afins ao evento.
Como palestrantes, foram convidadas mulheres da rede de mMFC que se destacavam pela atuação em pesquisa e discussão dos temas; pesquisadoras de outras áreas da saúde e ciências humanas; e ativistas dos coletivos 'Me farei ouvir' e 'Elas na política', ambos sobre a participação das mulheres na política, e da 'Casa Frida' (coletivo de cultura), 'Escola de Almas Benzedeiras de Brasília' (benzimento) e 'Gira Cerrado' (dança circular).

Não houve qualquer tipo de patrocínio ou financiamento externo para a realização do evento, respeitando a política de não financiamento por indústria farmacêutica da SBMFC. Todos os custos foram pagos com o repasse anual que o GT-MMFC recebe da SBMFC e com o valor das inscrições pagas pelas participantes graduadas e pós-graduadas. A maioria das palestrantes arcou com suas despesas de passagem e hospedagem por financiamento próprio. Estudantes da UnB não pagaram por sua inscrição, o que representou um pressuposto ético na construção do evento para as organizadoras.

A inscrição foi realizada on-line, por meio de um site para eventos, e a comunicação, feita pelo e-mail do GT-MMFC. A divulgação foi feita pela assessora de imprensa da SBMFC. A identidade visual foi criada, voluntariamente, por uma designer que apreciou o projeto do encontro.

Aos estudantes da UnB, foi ofertada a possibilidade de participarem como monitores e monitoras, com a devida certificação, e 15 estudantes contribuíram. Em cada sala de atividades, dois monitores ficaram responsáveis pela organização, teste multimídia, monitoramento do tempo, organização das perguntas e demandas relacionadas com a palestrante (recepção, certificado e agradecimentos). Os registros do evento foram realizados por uma fotógrafa local.

Torna-se substancial ressaltar o investimento da organização para possibilitar o 'Espaço brincar' e garantir a participação e inclusão das mulheres mães. Este contou com estrutura e brinquedos disponibilizados, em parte, pela comissão organizadora e, em parte, com o financiamento das inscrições, e cuidado 
das monitoras. O espaço público ainda carece de ambiência para que as mulheres possam utilizá-lo com ou sem os seus filhos, de forma articulada às suas atividades laborais ou acadêmicas. O enfrentamento das desigualdades de gênero na vida pública compõe a luta feminista em prol da democracia: trata-se de uma "revolução necessária no cotidiano das mulheres"8(5). Outra preocupação nesse sentido foi a oferta voluntária de hospedagem solidária para mulheres vindas de outras cidades e estados, ação baseada na colaboração genuína entre mulheres.

Além das atividades científicas, o Encontro proporcionou área de exposição de produtoras locais, momentos culturais, lanches e confraternização, todos os serviços fornecidos por empresas de mulheres. Houve apresentação das 'Filhas d'Oyá', grupo percussivo de mulheres do Distrito Federal, de Martinha do Coco, mestra de coco do Paranoá; sarau com autoras do livro 'Causos Clínicos - Histórias da Medicina de Família e Comunidade'; oficina de danças circulares ‘Gira Cerrado’ e bênçãos da 'Escola de Almas Benzedeiras de Brasília'. Essas iniciativas, focadas na economia solidária, buscaram estimular a contribuição, a renda e a inclusão das mulheres, historicamente imersas na assimetria sexual do trabalho do sistema capitalista ${ }^{10}$.

Figura 1. Comissão organizadora do I Encontro do GT Mulheres na MFC

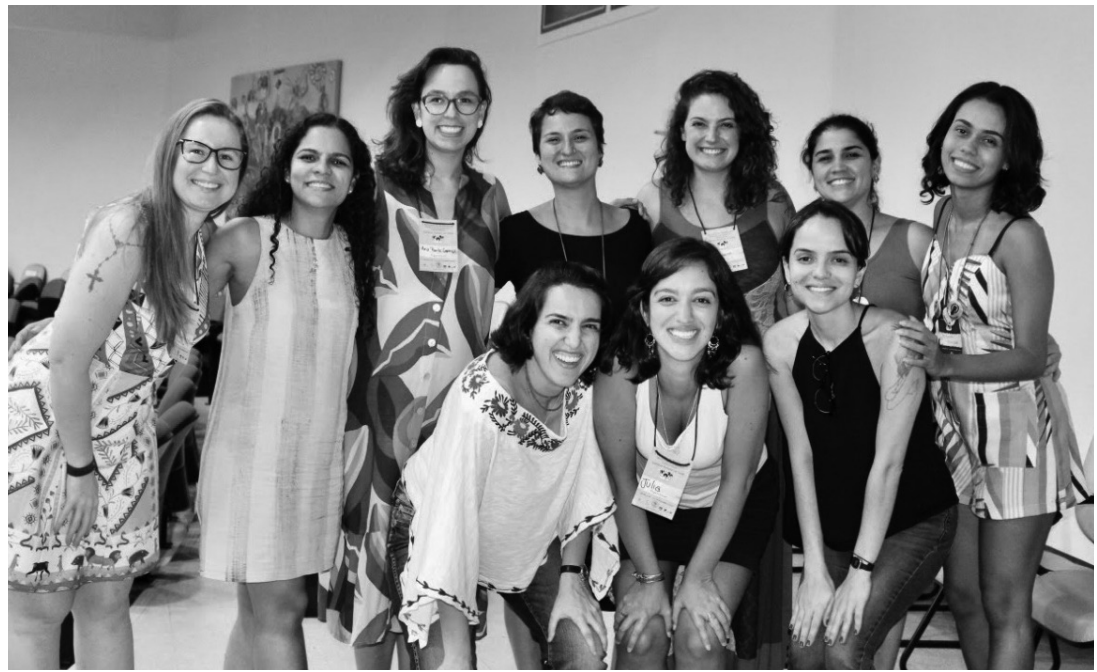

Fonte: Arquivo pessoal das autoras

Nota: Da esquerda para a direita, de pé: Ana Carolina, Denize Ornelas, Ana Paula Carrijo, Victoria Mey, Daiane Chavez, Carolina Reigada, Fernanda Fraissat. À frente, da esquerda para a direita: Patrícia Chueiri, Julia Horita, Camila Damasceno.

\section{A realização do encontro: participantes, atividades e discussões}

O evento foi organizado e efetivado por mMFC, de $1^{\circ}$ a 3 de novembro de 2019. Cento e trinta pessoas se inscreveram: apenas 6 eram do gênero masculino, 56 eram residentes ou estudantes da área da saúde. A média de idade das participantes foi de 33 anos. Grande parte das participantes era do Centro-Oeste ( $n=96)$, e o restante contemplou os estados do Sul, Sudeste e Nordeste. Destaca-se a ausência de mulheres 
da região Norte, o que o GT-MMFC identifica como um problema de representatividade e alcance da rede de mulheres, ainda sem uma solução estruturada. Apesar do enfoque na participação de mMFC, felizmente, profissionais de outras áreas participaram, como doulas, psicólogas, assistentes sociais e nutricionistas.

A grade de atividades é apresentada no quadro 1.

\begin{tabular}{ll}
\hline Quadro 1. Atividades científicas do I Encontro do GT Mulheres na MFC & \\
\hline Mesas-Redondas & Rodas de conversa e práticas \\
\hline Abertura: Protagonismo feminino - olhares & Mulheres na política \\
Maternagens, trabalho e decolonialidade & Como me cuido? Estratégias e dificuldades \\
Políticas públicas pela equidade e integralidade de mulheres trans e & Media training para mulheres \\
lésbicas & \\
Lidando com machismo, racismo e assédio na universidade & Como fazer pesquisa científica? \\
Gênero e a feminização da medicina & Interseccionalidades e rede de colaboração \\
& entre mulheres \\
Comunicação, mulheres e assertividade & Mulheres cuidando de mulheres: saberes tradi- \\
& cionais femininos e PICs \\
\hline
\end{tabular}

Fonte: Elaboração própria.

A partir do tema central, 'liderança feminina', as atividades realizadas podem ser divididas em três grandes ramos. No primeiro, estão aquelas voltadas para a reflexão e instrumentalização das participantes em assuntos relacionados com o desempenho da liderança em diferentes áreas, como as rodas de conversa 'Mulheres na política', 'Políticas públicas pela equidade e integralidade de mulheres trans e lésbicas', 'Media training para mulheres', 'Como fazer pesquisa científica' e 'Comunicação, mulheres e assertividade'. O tema do protagonismo feminino foi o mais prevalente nas respostas ao formulário enviado. $\mathrm{O}$ interesse pelo tema nos parece ser fruto do trabalho do GT-MMFC, pela coordenação de oficinas sobre liderança feminina em eventos regionais e nacionais da SBMFC, que foram citadas como o gatilho reflexivo para algumas mMFC. Estas começaram a repensar sobre sua posição no trabalho, interessando-se por questões como: iniquidade de gênero em posições de liderança em suas atividades laborais (inclusive dentro da própria SBMFC); assertividade; e como reconhecer e lidar com a chamada 'síndrome da impostora'.

Para a discussão sobre mulheres no exercício político, convidamos os coletivos 'Elas na política' e 'Me farei ouvir', que trabalham pela conscientização, motivação e capacitação de mulheres para a candidatura e protagonismo nos espaços de poder político. Segundo o mapa Mulheres na Política 201911, apesar de as mulheres comporem $52 \%$ da população, o Brasil tem uma das mais baixas representatividades femininas no governo, com apenas $15 \%$ de mulheres, com decréscimo para $9 \%$ a partir do governo de Jair Bolsonaro. Na SBMFC, no âmbito da história institucional, a iniquidade de gênero no conselho diretor e na presidência da sociedade era marcante. O GT-MMFC atua questionando essa baixa representatividade, assim como favorecendo a equidade de gênero na construção dos eventos científicos. O conselho diretor vigente tem 7 homens e 6 mulheres, e a paridade de gênero foi pauta da última eleição para diretoria da associação, o que nos parece efeito direto das ações do GT-MMFC. 
Na mesa sobre políticas públicas para mulheres trans e lésbicas, tivemos a participação da Dra. Tatiana Lionço, que trouxe o caminho percorrido para construção de uma política nacional12 no âmbito da saúde, a relevância dos direitos conquistados e a necessidade de mantê-los; e Láris, homem transexual que, sob as linhas da narrativa cartográfica, apresentou seu processo de transexualização e afetos em meio às suas relações pessoais, família e trabalho.

Para a prática sobre media training, a assessora de imprensa da SBMFC, Ana Carolina D'Angelis, explicou e demonstrou como ter mais influência e visibilidade nas redes sociais - principal ferramenta midiática de divulgação na contemporaneidade.

Na roda de conversa sobre pesquisa científica, as doutoras Patrícia Chueiri e Magda Oliveira trouxeram dados nacionais e mundiais sobre diferenças relacionadas com o gênero dentro da pesquisa científica, como o prejuízo para mulheres no total de financiamentos recebidos, menor reconhecimento no meio e maior dificuldade em publicar $\operatorname{artigos}^{13}$. Na área da MFC, apenas 12\% dos médicos de família e comunidade têm título de mestrado; e 2,7\%, de doutorado. O recorte de gênero sobre essa análise revela que as mMFC têm menores chances de obtenção dos títulos, principalmente o doutorado ${ }^{\mathbf{1 4}}$, o que nos parece um sintoma a ser analisado, pois, no Brasil, especialmente na área da saúde, mulheres pesquisadoras são maioria (60\%), e $49 \%$ das publicações têm mulheres como autoras $^{15}$. Como encaminhamento, foi criado um grupo de e-mails específico para pensar a produção científica feminina e possibilitar apoio, troca de experiências e parcerias entre pesquisadoras.

Na roda de conversa sobre comunicação, Jéssica Leão trouxe a importância do exercício da assertividade e da comunicação não violenta como ferramentas para expor claramente ideias e opiniões, afastando-se da representação social de mulher assertiva e protagonista como violenta. Trouxe a discussão sobre o 'machismo discursivo', que, por meio da linguagem, perpetua o machismo cotidiano ${ }^{16}$.

O segundo ramo temático abordou as dificuldades que mulheres encontram em diferentes ambientes de estudo e trabalho para o exercício do protagonismo. Neste, estão 'Maternagens, trabalho e decolonialidade' e 'Lidando com machismo, racismo e assédio na universidade'.

Na mesa-redonda 'Maternagens, trabalho e decolonialidade', a antropóloga doutora Marianna Holanda, a nutricionista doutora Renata Monteiro e a MFC mestra Natália Albuquerque discutiram as dificuldades enfrentadas pelas mulheres no ambiente de trabalho durante o ciclo gravídico-puerperal e a amamentação de seus filhos. Marianna discorreu sobre decolonialidade e a necessidade de complementar o feminismo tradicional e produtivista, proeminentemente branco, com as lutas antirracistas e indígenas, uma vez que ainda temos uma cultura escravocrata e excludente. Renata trouxe como a visão da amamentação pelos profissionais de saúde é ainda extremamente técnica e até violenta com as mulheres, focando na criança em detrimento delas. Natália expôs como gênero e maternidade se interseccionam e impactam nas trajetórias profissionais de $\mathrm{mMFC}$, discutindo os atravessamentos de questões sobre ética, responsabilização pelo cuidado, equilíbrio de vida pessoal, familiar e laboral17.

$\mathrm{Na}$ roda de conversa sobre machismo, racismo e assédio na universidade, a doutoranda Ana Paula Carrijo e as alunas de medicina da UnB Letícia Resende e Marina Moreira problematizaram sobre o assédio às mulheres em diversas instituições, como reconhecê-lo e medidas para proteção e enfrentamento.

No terceiro ramo temático, estão as atividades voltadas ao autocuidado e às possibilidades de cuidado entre mulheres, como as rodas de conversa 'Como me cuido: estratégias e dificuldades', em que a doutoranda Débora Teixeira trouxe teoria e vivência de mindfullness; 'Interseccionalidade e rede de colaboração de mulheres', em que as mestras 
Rita Helena Borret e Thamiris Oliveira trouxeram a necessidade do reconhecimento das diferentes opressões sofridas pelas mulheres negras, pobres, lésbicas, com embasamento teórico de autoras negras e lésbicas, reforçando o conceito de dororidade ${ }^{18}$. Os grupos 'Gira Cerrado', 'Escola de Almas Benzedeiras de Brasília' e 'Coletivo Frida' contribuíram, respectivamente, com reflexões e práticas sobre dança circular, benzimento e doulagem, em atividades muito elogiadas pelas participantes, que puderam vivenciar o potencial do cuidado.

Na mesa de abertura, estiveram as doutoras Maria Inez Padula Anderson e Valéria Mendonça e a mestra Natália Albuquerque. Maria Inez foi convidada por sua trajetória relevante como MFC, tendo desempenhado diferentes papéis de liderança - no Departamento de Medicina Integral, Familiar e Comunitária da Universidade do Estado do Rio de Janeiro (Uerj), na Confederação Iberoamericana de Medicina Familiar e na própria SBMFC -, e trouxe suas experiências e aprendizados. Valéria discutiu a conscientização sobre posturas e atitudes, em uma leitura intencional e coerente da realidade, como forma de buscar propósitos com a lucidez do espaço que ocupamos no meio social. Natália foi convidada com o intuito de partilhar a experiência de trajetória profissional de uma jovem médica, coordenadora de programa de residência médica e pesquisadora.

Já na mesa de encerramento, a doutora Valeska Zanello abordou a saúde mental das mulheres, os dispositivos de gênero ${ }^{19} \mathrm{e}$ a necessidade da conscientização de seu papel e atuação de mulheres, especialmente as profissionais de saúde. A mestra Mariana Paes refletiu sobre os lugares de fala e desafios da expressão de subjetividades relacionadas com as iniquidades de gênero ${ }^{20}$.

A mestra Denize Ornelas desvelou a feminização da medicina ${ }^{\mathbf{2 1} \mathbf{2 2}}$, os desdobramentos percebidos nas pesquisas quanto à (des) valorização social do trabalho médico com o avanço do número de mulheres médicas em outros países ${ }^{\mathbf{2 3} 24}$, a assimetria de gênero no poder e cargos de chefia, as diferenças na formação e na escolha pelas especialidades médicas e, consequentemente, a diferença de remuneração média, mais baixa entre as mulheres ${ }^{25}$, mesmo quando corrigida a carga horária, mantendo-se um desafio a equidade no âmbito econômico da profissão. Essa mesa trouxe questões como incluir novas mMFC no debate dos temas aqui evidenciados e repensar o papel do GT-MMFC nessa articulação.

\section{Avaliação do evento e desdobramentos}

Durante a reunião do GT-MMFC, ocorrida no último dia do encontro, as integrantes avaliaram que o evento alcançou os objetivos iniciais de aprofundar discussões despontadas no congresso da SBMFC e reunir mulheres de diferentes partes do Brasil, divulgando o GT-MMFC ${ }^{26}$. Alguns encaminhamentos, como ampliar intervenções do GT-MMFC dentro e fora da SBMFC, estão sendo viabilizados com publicações de notas e vídeos sobre temas de interesse científico e político ${ }^{5}$.

O grupo avalia que, progressivamente, mais mulheres vêm participando das discussões científicas nos espaços providos pela SBMFC, o que potencializa transformações institucionais. Exemplificando, o GT-MMFC propôs a atualização da política editorial da 'Revista da Sociedade Brasileira de Medicina de Família e Comunidade' (RBMFC) para a inclusão de mulheres nos cargos de edição da revista, o que desencadeou a integração de duas mMFC desde setembro de 2020.

Em 2020, com as eleições para a diretoria da SBMFC, percebeu-se um movimento ativo das chapas em contemplar discussões trazidas pelo GT-MMFC, não só sobre paridade de gênero, mas também sobre aborto legal. Entendemos que tais desdobramentos têm ligação direta com a atuação do GT-MMFC em diferentes espaços, tanto operando sobre iniquidades de gênero, violência de gênero na prática clínica e no desempenho profissional 
de mMFC quanto por intermédio de mulheres líderes fomentando tal discussão. Esse encontro nos pareceu essencial para apontar as iniquidades e a urgência de ampliarmos as discussões do GT-MMFC nos espaços científicos da especialidade.

Outra fortaleza é a colaboração entre os GT da SBMFC, principalmente com o GT de Gênero, Sexualidade, Diversidade e Direitos e o GT de Saúde da População Negra, aprofundando temas relacionados não só com a interseccionalidade, mas construindo verdadeiras redes de colaboração, já que muitas pautas são transversais às discussões feitas nesses três GT, potencializando as exigências e aprofundando as discussões.

Entre os problemas percebidos, não houve a adesão de médicos de família e comunidade, de representantes da diretoria da SBMFC e da associação estadual ao Encontro. Ressalta-se que a participação de indivíduos do gênero masculino não estava vedada, porém a divulgação do evento dirigiu-se ao público usando artigos femininos, o que pode ter gerado dúvidas e incômodos sobre a participação masculina. Ademais, observou-se o escasso interesse dos homens nos temas abordados, com foco em questões relativas à saúde e atuação política das mulheres. Compreendemos que essa baixa adesão reflete o desinteresse dos homens sobre as pautas relacionadas com o posicionamento político das mulheres, para além de sua saúde biológica, mantendo-se afastados desse debate. Apesar de ressaltarmos o protagonismo feminino, consideramos estrutural a interlocução com os homens, para discutirmos e transformarmos juntos a sociedade científica e a prática clínica da MFC.

Alguns problemas organizacionais foram apontados, como a dificuldade de conseguir financiamento para o evento, a não divulgação prévia dos locais de confraternização; a falta de um canal de comunicação dinâmico durante a realização do Encontro (talvez um aplicativo do evento) e a quantidade de plástico utilizada nos lanches dos intervalos. Também foi levantada a pouca participação de $\mathrm{mMFC}$ da própria cidade, a ínfima participação da associação regional da especialidade e a presença de programação concorrente com a reunião final do GT-MMFC, que dividiu as participantes entre os espaços, deixando de oportunizar a ocasião como um momento de fortalecimento da rede de mMFC e do próprio GT-MMFC.

Como sugestões, trouxeram estimular o consumo consciente de plásticos; divulgar melhor o mapa das salas; incluir a possibilidade de exposição de trabalhos científicos de mulheres; aumentar as atividades em conjunto com outros GT e prezar pela participação de uma mMFC em todas as mesas do congresso, trazendo a visão da especialidade para a temática debatida.

Quanto às fortalezas percebidas no Encontro, citaram-se: a gratuidade para alunos da UnB; as atividades culturais; a diversidade de temas; a forma participativa de montar a programação; as monitoras e monitores; a valorização das palestrantes locais; as atividades de cuidado e autocuidado; e o 'Espaço de brincar'. Esses e outros aspectos organizacionais foram buscados de forma intencional pela comissão de organização do Encontro, levando em conta a $\mathrm{GES}^{6}$, os 10 passos resumidos para Equidade de Gênero para Encontros Científicos da Wonca, 2010 (quadro 2) 1.

A GES orienta que os encontros científicos busquem: formas de apoiar a participação de mães, pais e cuidadores provendo, por exemplo, espaços de cuidado e suporte às crianças; o convite intencional de mulheres palestrantes; o estímulo e desenvolvimento de lideranças; a promoção de atividades sociais e a busca de patrocinadores que respeitem gênero, origem nacional e etnicidade; o uso de 'bolsas' para estímulo à participação dos encontros. A comissão considera que a maioria desses itens foi cumprida de forma integral e avalia ainda que propôs formas alternativas, diante das restrições financeiras enfrentadas, de se aproximar do ideal colocado, com a isenção de taxas de inscrição e a participação de monitores com certificação. Os únicos dois itens das 
GES que a comissão não buscou ativamente foi ter o equilíbrio de gênero no Encontro e na própria comissão de organização. A comissão avaliou que o momento histórico de organizar um primeiro encontro de mMFC, feito pelo esforço e colaboração das próprias mulheres, suplantou a orientação de haver uma paridade de gênero no evento. Porém, como já explicitado, é importante que um próximo passo seja dado nessa direção.

Quadro 2. Dez passos resumidos para equidade de gênero para encontros científicos da Wonca, 2010

1. Estrutura das Comissões: todas as Comissões envolvidas no planejamento e convocação de reuniões científicas aderem aos princípios básicos de equilíbrio de gênero e equidade de gênero.

2. Conteúdo da programação: incorpora a igualdade de gênero ao longo de sua implementação. Todos os temas das reuniões científicas incluem uma perspectiva ou análise de gênero. Todos os convites à apresentação de propostas para o plenário, simpósios e workshops solicitam explicitamente uma consideração de gênero. Sem restrições/viés de gênero na participação. Os temas relacionados à saúde das mulheres são incentivados, independentemente do tema do encontro.

3. Equilíbrio de gênero: todas as comissões científicas planejam o equilíbrio de gênero para oradores/palestrantes convidados para plenárias, oficinas e simpósios.

4. Palestrantes: mulheres merecedoras são propositalmente e proativamente consideradas como palestrantes

5. Bolsas de estudo: a Comissão de Organização ou o comitê de inclusão por bolsas emprega todos os esforços possíveis para aumentar propositadamente o conjunto de fundos disponíveis e distribuir bolsas equitativamente em relação ao gênero.

6. Desenvolvimento da liderança: as Comissões Científicas empreendem todos os esforços possíveis para promover o desenvolvimento da liderança em cada conferência.

7. Atividades da família: a Comissão de Organização colabora com os participantes que são mães, pais ou cuidadores de crianças, a fim de facilitar os arranjos necessários para oferecer cuidado acessível para bebês, crianças pequenas e crianças em idade escolar.

8. Eventos sociais: a Comissão de Organização garante que todas as atividades sociais oferecidas como parte do programa regular da conferência respeitam gênero, origem nacional e etnicidade dos participantes e seus convidados, e que humor ou eventos sexistas e/ou comentários degradantes não serão tolerados.

9. Patrocínio e marketing corporativo: o Comitê Científico que organiza os encontros da Wonca restringe os patrocinadores externos que conduzem políticas ou comercializem produtos que afetam negativamente as mulheres. Todo esforço é feito para restringir imagens ou produtos que objetificam as mulheres ou que fazem alegações enganosas.

10. Estilos educativos interativos: as Comissões Científicas incentivam os palestrantes convidados a adotar estilos de ensino e de aprendizagem interativos e incluem esse encorajamento nos convites à apresentação de propostas para cada reunião.

Fonte: Oliveira, Chueiri, Albuquerque ${ }^{1}$

A pandemia da Covid-19 interrompeu alguns projetos programados e em desenvolvimento, como o II Encontro do GT-MMFC com realização prevista no Congresso Nordestino de MFC, que ocorreria em julho 2020; e uma campanha que vinha sendo desenvolvida, durante o mês de março, pelo protagonismo feminino em diversas áreas. Algumas campanhas, para aumento da consciência de gênero no cotidiano e sobre formas de apoio entre mulheres para aumento da pesquisa e publicação acadêmica, ainda não foram colocadas em prática.

Outra repercussão foi o fortalecimento do GT-MMFC ante os demais GT da sociedade científica, mas, principalmente, perante sua diretoria, que parece reconhecer o potencial de trabalho do grupo e o valor que ele agrega à SBMFC. 


\section{Conclusões}

Nos últimos quatro anos, o GT-MMFC vem amadurecendo suas ações de forma propositiva, avançando com a inclusão crescente de mMFC e estudantes de medicina. Em junho de 2021, há aproximadamente 60 mulheres cadastradas e adimplentes participando ativamente das decisões e organização das atividades do GT, e 120 mulheres no grupo de acolhimento, no qual acontecem discussões mais amplas. Observamos que sua atuação vem preenchendo e problematizando a lacuna da consciência de gênero, com clara influência no cotidiano de médicas e médicos de família e comunidade, na prática ambulatorial, acadêmica, científica e da gestão.

O I Encontro do GT-MMFC consolidou a experiência acumulada e fortaleceu o grupo para progredir de forma abrangente, diversa e crítica. Nesse sentido, este relato tem importância histórica e documental para as mMFC e, de forma mais ampla, para os movimentos de mulheres na medicina e na saúde, no sentido de materializar a importância das pautas elegidas. Ainda existem amplos desafios, como a pouca representatividade no GT-MMFC de mulheres do Nordeste e do Norte, a ínfima participação de homens nas discussões levantadas e o enfrentamento do machismo e da assimetria de gênero na medicina. Espera-se que o grupo continue a advogar para que mais profissionais reconheçam a questão de gênero como um condicionante social de saúde e transformem sua práxis em prol do enfrentamento das iniquidades.

\section{Colaboradoras}

Reigada CLL (0000-0001-9621-4908)*, Oliveira DOPS (0000-0001-8809-0095)*, Carrijo APB (0000-0002-4220-6109)*, Chueiri PS (0000-0002-0811-3910)*, Moherdaui JH (0000-0001-9167-3937)* e Albuquerque NP (0000-0001-6055-1515)* contribuíram igualmente para a elaboração do manuscrito.

\section{Referências}

1. Oliveira DOPS, Chueiri PS, Albuquerque NP. Carta de Cuiabá - Mulheres, Médicas de Família e Comunidade, no Brasil - onde estamos e onde podemos chegar? Rev Bras Med Fam Comunidade. 2020; 15(42):1784.

2. Scheffer M, coordenador. Demografia Médica no Brasil 2018. São Paulo: Departamento de Medicina Preventiva da Faculdade de Medicina da USP; Conselho Regional de Medicina do Estado de São Paulo; Conselho Federal de Medicina; 2018. [acesso em
2020 jul 20]. Disponível em: https://cdn-flip3d.sflip. com.br/temp_site/edicao-97e48472142cfddlcd5d5b5ca6831cf4.pdf. de Janeiro: Nova Fronteira; 2016.

4. Rohden F. Uma Ciência da Diferença: sexo e gênero na medicina da mulher. Rio de Janeiro: Editora Fiocruz; 2001.
*Orcid (Open Researcher and Contributor ID). 
5. Pontes N, Chueiri P, Ornelas P. Grupo de Trabalho Mulheres na MFC: Histórico e atividades Nov 2017 - Jul 2019. Cuiabá: CBMFC; 2019. [acesso em 2020 jul 6]. Disponível em: https://www.sbmfc.org.br/wp-content/uploads/2019/08/Historico-e-atividades-GT-Mulheres-2017-19.pdf

6. Wonca Working Party on Women and Family Medicine. Gender equity standards for Wonca Scientific Meetings. East Anglia: WWPWFM; 2009. [acesso em 2020 jul 6]. Disponível em: https://www.globalfamilydoctor.com/GetFile.aspx?oid=C34EDB0E-B59C-4D4B-A518-09250FA34B7A.

7. Sociedade Brasileira de Medicina de Família e Comunidade. Ata da Reunião Ordinária do Grupos de Trabalho Mulheres na MFC. Cuiabá: CBMFC; 2019. [acesso em 2020 jul 6]. Disponível em: https://www. sbmfc.org.br/wp-content/uploads/2019/08/Ata-Reuniao-Gt-Mulheres-15CBMFC.pdf.

8. Machado LZ. Feminismos brasileiros nas relações com o Estado: contextos e incertezas. Cadernos pagu. 2016; (47):e16471.

9. Universidade de Brasília. Diretoria da Diversidade. [acesso em 2020 jul 10]. Disponível em: http://diversidade.unb.br/index.php/noticias/177-video-institucional-da-div-dac-unb.

10. Bonumá H. As mulheres e a economia solidária: a resistência no cotidiano tecendo uma vida melhor. [monografia]. Porto Alegre: Universidade Federal do Rio Grande do Sul; 2015.

11. Câmara dos Deputados. Política e administração pública: Baixa representatividade de brasileiras na política se reflete na Câmara. [acesso em 2020 jul 13]. Disponível em: https://www.camara.leg.br/noticias/554554-baixa-representatividade-de-brasileiras-na-politica-se-reflete-na-camara/.

12. Brasil. Ministério da Saúde. Política Nacional de Saúde Integral de Lésbicas, Gays, Bissexuais, Travestis e Transexuais - LGBT. Brasília, DF: Ministério da Saúde; 2008.
13. Kellogg School of Management at Northwestern University. How Big Is the Gender Gap in Science Research Funding? [acesso em 2020 jul 20]. Disponível em: https://insight.kellogg.northwestern.edu/article/how-big-is-the-gender-gap-in-science-research-funding.

14. Fontenelle LF, Rossi SV, Oliveira MHM, et al. Postgraduate education among family and community physicians in Brazil: the Trajetórias MFC project. medRxiv preprint first posted online Oct 5, 2019. [acesso em 2020 jul 20]. Disponível em: https://www.medrxiv.org/content/10.1101/19005744v2.

15. Prusa A, Picanço L, editores. A Snapshot of the Status of Women in Brazil: 2019. Brazil Institute. Washington, DC: Wilson Center; 2019. [acesso em 2020 jul 20]. Disponível em: https://www.wilsoncenter. org/sites/default/files/media/documents/publication/status_of_women_in_brazil_2019_final.pdf.

16. Barros AT, Busanello E. Machismo discursivo: modos de interdição da voz das mulheres no parlamento brasileiro. Rev. Estud. Fem. 2019 [acesso em 2020 jul 20]; 27(2). Disponível em: http://www.scielo.br/ scielo.php?script=sci_arttext\&pid=S0104-026X201 9000200219\&lng=pt\&nrm=iso.

17. Albuquerque NP. Trajetórias de vida, marcadores de diferença e as escolhas, caminhos e permanência de médicas de família e comunidade na estratégia saúde da família. [dissertação]. Uberlândia: Universidade Federal de Uberlândia; 2019. 158 p. [acesso em 2020 jul 20]. Disponível em: http://dx.doi.org/10.14393/ ufu.di.2019.2222.

18. Piedade V. Dororidade. São Paulo: Nós; 2017.

19. Zanello V. Saúde mental, gênero e dispositivos: cultura e processos de subjetivação. Curitiba: Appris; 2018.

20. Paes MF. Mulheres gestoras e trabalhadoras no SUS: reflexões sobre seus lugares de fala e os desafios para expressão de subjetividades. In: Conceição HRM, Túlio Batista Franco, organizadores. Cartografias na 
saúde: ensaios da multiplicidade no cuidado. Porto Alegre: Rede UNIDA; 2018.

21. Scheffer MC, Cassenote AJF. A feminização da medicina no Brasil. Rev Bioét. 2013 [acesso em 2020 jul 20]; 21(2):268-77. Disponível em: http://dx.doi. org/10.1590/S1983-80422013000200010.

22. Scheffer MC, coordenador. Departamento de Medicina Preventiva, Faculdade de Medicina da USP. Conselho Regional de Medicina do Estado de São Paulo. Demografia Médica no Brasil 2015. São Paulo: CFM; 2015. 284 p.

23. Wallace AE, Weeks WB. Differences in income between male and female primary care physicians. J American Med Women's Assoc. 2002; 57(4):180-4.

24. Harden J. 'Mother Russia' at work: gender divisions in the medical profession. Europ. J. Women's Studies. 2001; 8(2):181-99.
25. Mainardi GM, Cassenote AJF, Guilloux AGA, et al. What explains wage differences between male and female Brazilian physicians? A cross-sectional nationwide study. BMJ Open. 2019; 9(4):e023811.

26. Ata Reunião Ordinária do Grupo de Trabalho Mulheres na MFC ocorrida no I Encontro do Grupo de Trabalho - Mulheres na Medicina de Família e Comunidade. Grupo de Trabalho - Mulheres na Medicina de Família e Comunidade. [acesso em 2020 jul 20]. Disponível em: https://www.sbmfc.org.br/wp-content/uploads/2020/07/Ata-Reuniao-Gt-Mulheres-I-Encontro.docx.pdf.

\footnotetext{
Recebido em 13/08/2020

Aprovado em 07/07/2021

Conflito de interesses: inexistente

Suporte financeiro: não houve
} 